\title{
Von der Lust an Sterbenssachen. Das Schreckliche lockt
}

»Es ist eine allgemeine Erscheinung in unserer Natur, dass uns das Traurige, das Schreckliche, das Schauderhafte selbst mit unwiderstehlichem Zauber an sich lockt.«

(F. Schiller, Über die tragische Kunst, 30)

Brigitte Boothe

Todeslust in der Literatur des 20. Jhs.

Das 20. Jh. der Weltkriege, der Demontage von Autoritätstraditionen und der säkularen Demokratisierung hat eine europäische Kunst und Literatur hervorgebracht, die reich ist an religiösen Motiven. Menschliche Existenz erscheint in komischer oder tragischer Fragilität. Sie kommt als beschädigt, als Schwundform, als todverfallen ins Bild. Komische Gottsuche, Gottesfinsternis und leere Transzendenz sind nicht nur Schlüsselthemen für Franz Kafka und Samuel Beckett. Menschliche Existenz in komischer und tragischer Fragilität und Todverfallenheit war auch ein wichtiges Thema der klassischen Psychoanalyse. Freud spricht vom »Todestrieb« (1920) als machtvollem und stumm wirksamem Drang, das eigene Absterben herbeizufuihren. Dieser Drang, sich selbst und andere auszulöschen, ist unter bestimmten krisenhaften Bedingungen mächtiger als der "Lebenstrieb", das Verlangen, Leben zu erhalten, zu vermehren, zu erneuern, zu erweitern. Ersteres, der "Lebenstrieb«, ist eine krafterfüllte Bewegung des Gedeihens, der Entwicklung, der Reife, der Individuation, der Selbstvollendung des Einzelnen oder einer Gemeinschaft. Letzteres, der »Todestrieb", eine von innen heraus wirksame Bewegung des gewaltsamen oder leisen Verlöschens, der unbedingten Preisgabe an die Destruktion. Diese evolutionär befremdliche Idee wirkt überaus phantasiebeflügelnd, wahrscheinlich gerade der bildhaften und vagen Formulierung wegen. Sie bleibt bis heute innerhalb und ausserhalb der Psychoanalyse umstritten. Sie regt indessen zum Verständnis an für eine breit gefächerte kulturelle Erscheinung des 20. Jhs., und zwar, wenn es, jenseits konfessionell bekennender Kunst und Literatur, um menschliche Bezogenheit auf ein Göttliches geht. Dann sind menschliches Nicht-Gedeihen, das Ausbleiben von Individuation und Personalität, Preisgabe an Tod und Verlöschen Schlüsselmerkmale der Darstellung. Sie verbinden sich auf abgründig ironische Weise mit einem prekären sakralen Bezug, zum Beispiel bei Kafka mit dem vergeblichen Warten auf den "Schlossherrn", das 
"Gericht«, das »Gesetz«, bei Samuel Beckett sprichwörtlich mit dem "Warten auf Godot«. Todestrieb als Abwendung von sozialer Integration, um sich zu verschleudern und auszulöschen und Teil einer grossen Leere zu werden, die etwas Sakrales hat.

\section{Messias-Anspielungen, Verlorene Söhne, Ungerettete}

Grossartiger Selbstzerfall ist todesbrünstige Hingabe an grossartig leere Transzendenz. "Heilige Trinker" und nirwanaverfallene Fixer, Depressionszermalmte und bindungslose "Taxidriver" sind uns aus Belletristik, Film und Fernsehen bis heute und bis zum Überdruss wohlbekannt. Diesen Geschichten ist gemeinsam, dass die - überwiegend männlichen - Protagonisten, verstrickt in ein selbstzerstörerisches Aktionsfeld, sich nicht entwickeln, sondern in radikaler Zurückgeworfenheit auf sich selbst in ihrer prekären Existenz jenseits sozialer Einbindung zerfallen, und dass dieser Zerfall eine Art Grosse Absage hin zu einer Art Grosser Freiheit vermittelt. Diese Dramaturgie bedient sich nicht selten explizit religiöser Bildtraditionen und Handlungskonstellationen. Eine davon soll im Folgenden exemplarisch zur Sprache kommen, eine messianische Dramaturgie. Es ist die Geschichte eines Sohnes, der vermeintlich und nach dem Willen des Autors F. Dürrenmatt auf lachhafte Weise zur heiligen Erlöserfigur wird. Diese Sohnesfigur, die als Messias auftreten soll, zeigt modellhaft die Verbindung von Entwicklungsstagnation, personalem Zerfall und vergeblicher Suche nach dem Grossen Vater. Diese Sohnesgeschichte ist interessant, weil sie eine innere Logik hat. Sie lässt sich studieren in Dürrenmatts späten und wenig bekannten Romanentwurf "Der Rebell« (Stoffe III, 281). Hier findet sich unverhüllt das grosse religiöse und literarische Motiv vom Messias, vom gottgesandten Menschheitserlöser. Dürrenmatt verweist im Titel »Der Rebell« freilich nicht auf den religiösen Kontext, sondern auf das Bedeutungsfeld des gesellschaftlichen Umsturzes. Dieser bestimmt aber nicht den Inhalt des Erzählwerkes. Vielmehr sind es dann in der Tat Motive der Befreiung, Rettung und Erlösung, wie die religiöse Überlieferung sie bietet. So ist der künftige »Rebell« zunächst ein verlassener, verarmter und bedürftiger Sohn, der den Vater sucht und schliesslich auf eine heilige Vater-Figur im Amt eines Hohepriesters trifft; ein bedrücktes, geknechtetes Volk hofft auf Erlösung durch einen verheissenen Heiland, man hält ihn, den vermeintlichen Rebellen, für den angekündigten Messias. 


\section{Der schwache Sohn als literarischer Protagonist}

König Ödipus ist ein starker Sohn und scheitert an seiner Stärke. Der Rebell ist ein schwacher Sohn und feiert das eigene Absterben: heilige Todeslust. Söhne sind es: der Rebell, der verlorene Sohn des Neuen Testaments, König Ödipus. Ödipus, der König, der Bezwinger der Sphinx, Befreier von Theben, Gatte und Vater, wird im Lauf der Ermittlungen ein anderer. Wir sehen auf der Bühne, wie dieser Sohn, der unwissentlich seinen Vater erschlagen hat, sich in einem zerreissenden Prozess zwischen Zweifel, Entsetzen und Ahnung der eigenen Geschichte stellt, sich das Augenlicht nimmt, blind ist wie Teiresias, der Seher, und in der Isolation der sozialen Ächtung weiterlebt als ein anderer und ein Wissender. Der verlorene Sohn, reich ausgestattet mit dem Gut, das der Vater ihm auf Verlangen zugeteilt hat, scheitert in der Welt, erfährt dort, verarmt und ruiniert, soziale Ächtung und verwandelt sich, indem er sein Scheitern und das Ausmass seines Scheiterns anerkennt und gerade darin die Liebe des Vaters als Segen und Rettung erfährt. Der Rebell aber - und damit wird er zu einem Modellfall moderner Literatur - verwandelt sich nicht. Er ist ein Immergleicher, existiert und verendet. Die Verwandlung ist ihm versagt, und damit gleicht er all den anderen Protagonisten der Dürrenmattschen Komödien und vieler grossen und weniger grossen Schriftsteller der Aktualität. Das Ausbleiben der Verwandlung ist bei Dürrenmatt komödienhaft; und doch fühlen wir Leser und Zuschauer uns kaum erheitert, denn alles, alles schreit nach Verwandlung. Sie bleibt aus.

\section{Versagende Eltern, verlassenes Kind: ein Erfolgsdrehbuch}

Freud analysierte in der Dramaturgie der Ödipustragödie ein zentrales Paradigma von Liebe und Macht. Er analysierte den emotionalen Mitvollzug des Zuschauers oder Lesers und formulierte auf dieser Basis das Drehbuch vom Liebesanspruch des starken Sohnes der Mutter gegenüber, vom Wunsch, den Vater auszuschalten und der furchtbaren Konsequenz, sich dem strafenden Überich auszuliefern. Freud legte in seiner Schrift "Jenseits des Lustprinzips" (1920) und in "Das ökonomische Problem des Masochismus" (1924) den Ansatz einer Geschichte, die uns Heutige mehr bewegt: Ein schwacher Sohn entgeht der Herrschaft eines todbringenden Überichs nicht, er betreibt selbstruinös und als ein systematisch Verfallender die eigene Auslieferung. Das ist ein thematisches Feld, das nicht nur 
im psychoanalytischen Denken des späten Freud, sondern auch im 20. Jh. in Kunst und Literatur Prominenz geniesst. Es war und ist noch in mannigfacher Ausgestaltung beherrschend präsent, auch für die Psychoanalyse: als Thematik der Vaterlosigkeit, der ungenügenden und versagenden Eltern, der vaterlosen Gesellschaft, der Selbstsuche und Vatersuche, als Thematik des Narzissmus und des Ringens um Identität - oder kabarettistisch mit Robert Gernhardt ausgedrückt: »ich ich ich«. Diese »narzisstische Krise« oder »Krise der Selbstwerdung« (von Matt 1999, 369) steht als ödipales Paradigma vom starken, aber schuldigen Sohn jenem vom bedürftigen und verlassenen Kind (Kohut 1971) gegenüber. Diese einfache Gegenüberstellung zeigt bei Ödipus auf den schuldigen Sohn und bei Narziss auf schuldige Eltern. Die Anklage der schuldigen Eltern ist prominent und beliebt, in der Literatur, der Publizistik und der Psychotherapie. Auch Dürrenmatt scheint diesem Schema - wenn auch tragikomödiantisch - im Romanentwurf »Der Rebell« gewissenhaft zu folgen. Er führt uns Lesern geradezu aufdringlich die sprichwörtlich kalte Mutter und den sprichwörtlich sich entziehenden Vater vor und das arme Kind, das an den schuldigen Eltern zugrunde geht.

\section{Liebe, die den Tod will}

Aber so einfach ist es nicht. Und Dürrenmatts Rezept auch nicht. Da gibt es ein Jenseits der Elternschuld. Und das muss uns interessieren. Das scheinbar bloss eindimensionale Paradigma von vergeblicher Identitätssuche in gott- und vaterloser Welt hat einen verborgenen Hintergrund. Erst wenn man diesen Hintergrund aus seiner Verborgenheit zieht und beleuchtet, erhält die Identitätssuche im Drehbuch ihren schlüssigen Ort, wird ihre Vergeblichkeit notwendig, die Elternanklage schlüssig. Dürrenmatts Romanentwurf »Der Rebell« ist zwar eine scheinbar unscheinbare Geschichte und einem grösseren Publikum wenig bekannt, aber sie eignet sich trotzdem, hier eine machtvolle erotisch-sexuelle Dramaturgie freilegen, die sich selbst zu verneinen sucht und aus der Verneinung heraus den Gang der Ereignisse tödlich und todbringend determiniert. Diese Dramaturgie einer Liebesfinsternis gestaltet sich als Labyrinth der Kälte und Unfruchtbarkeit, als Offensive der Verachtung und der Todeslust. In dieser Programmatik der Todeslust finden sich ratio als Rationalität, die in aller Schärfe das "Schlimmstmögliche« artikuliert, und religio als heiliger todbringender Kosmos. 
"Eine Geschichte ist dann zu Ende gedacht, wenn sie ihre schlimmstmögliche Wendung genommen hat", so lautet der dritte von "21 Punkte(n) zu den Physikern" (Dürrenmatt 1962, 82). Die Geschichte vom Rebellen wendet sich von der Verlassenheit des Sohnes zur bodenlosen Verlassenheit in Gefangenschaft, die Vatersuche scheitert, die Bedrückung des Volkes setzt sich fort, das Religiöse ist Farce. Es ist die Geschichte eines Sohnes, den man radikal im Stich lässt und verrät, der gerade nicht wie der verlorene Sohn des Neuen Testaments Schutz und liebevolle Aufnahme beim Vater findet. Dieser Rebell ist ein Jüngling ohne Gesicht. Er sitzt nach dem Willen seines Autors als lächerliche Existenz im Labyrinth gefangen, um dort zu verenden. Warum kommt es nicht zur rettenden Begegnung von Vater und Sohn? Weil der Sohn für wirkliche Begegnungen und wirksames Eingreifen in dieser Welt verloren ist. Er ist verloren, weil er im abgeschiedenen und verborgenen Liebesreich der Mutter gefangen ist. Denn die Geschichte von der vergeblichen Vatersuche hat ihre Basis nicht im Verlangen des Sohnes, mit dem Vater zu ringen, konfrontativ, rebellierend, anerkennend, reibungslustig. Sie hat ihre Basis vielmehr in jenem Liebeslabyrinth, gerade weil der Text es lediglich anlegt - wenn auch in aller Konsequenz und keineswegs offen und transparent artikuliert. Eindrucksvoll und schlüssig organisieren die an dieses mütterliche Liebesreich sich knüpfenden Angst- und Lustphantasien den besonderen Gange der Dürrenmattschen Erzählung und lassen sie zu einem Modellfall jener 'Lust an Sterbenssachen Kultur des 20. Jhs. sie gestaltet hat.

\section{Ein Exodus in den Tod}

Dürrenmatts Romanentwurf „Der Rebell« ist die Geschichte eines Sohnes, der seine Heimat verlässt und in die Welt hinauszieht, um seinen Vater zu suchen. Damit entfaltet der Autor eine Erzähldynamik, die einen Exodus gestaltet, nicht den Exodus eines Volkes wie beispielsweise im biblischen Exodus der Israeliten aus Ägypten, nicht den Exodus ins Abenteuer, in die weite Welt, wie beispielsweise bei "Hänschen klein", sondern den Exodus, der als Suche nach dem Ursprung angelegt ist. Der Exodus des Kindes - seine Chance zur Freiheit und zur Positionierung in der Welt - ist ein Prüfstein der Macht und der Liebe (Boothe 1999). Alle Geschichten, die diese existentielle Situation thematisch konstellieren, lassen sich in einem 
Koordinatensystem anordnen, das die damit verknüpften Erfahrungen bündelt. Die Heimatbasis, gewöhnlich in Gestalt der Mutter oder des Vaters oder beider Eltern in ihrem Umfeld, stellt eine Ressourcenbasis dar. Sie kann reich oder dürftig sein. Die karge Heimat oder die bösen Eltern können den Exodus erzwingen, dann wird er zur Flucht oder zur Notemigration wie häufig im Märchen. Die reiche Heimat hingegen kann unstillbares Heimweh motivieren, aber auch Selbstvertrauen schaffen, weil die Eltern das Kind reich für die Reise ausstatten - als Repräsentanten einer Dynastie gleichsam und weil es nicht als Bettler, sondern als Botschafter einer guten Welt in der Fremde auftritt. Aber dieser materielle oder geistige oder emotionale Reichtum kann die Eltern zu unüberwindlichen Monumentalgestalten machen, denen man in kindlicher Unterwerfung treu bleibt wie der zur starken Monumentalgestalt des gütigen Vaters zurückgekehrte verlorene Sohn. Der Exodus kann mit elterlichem Kredit oder elterlichem Misskredit erfolgen, mit Fluch oder mit Segen. Das Kind kann in der Ferne den Eltern Ehre machen oder Schande bereiten. Der Exodus gestaltet die Generationenspannung von Verfall und Gedeihen. Im Gleichnis vom verlorenen Sohn ist der Sohn dem Verfall preisgegeben, er scheitert vollständig am Versuch, autonom zu werden, alles Gedeihen ist beim Vater, alle Ressourcen des Lebens sind beim Vater, der Preis der Rettung durch den Vater ist Autonomieverzicht. Vom Väterlichen gehen das Leben und die Ordnung des Lebens aus. Anders gestaltet sich das bei Joseph und seinen Brüdern. Da verfügt der Sohn über Ressourcen der Macht, der Stärke, des Reichtums. Da kann der ehemals von den Brüdern Bedrohte überlegene Versöhnungsbereitschaft auf der Basis der Machtumkehr geniessen. Die Generationenspannung kann sich aber auch im Schatten einer Überlebensschuld entfalten: Wenn die Söhne und Töchter leben dürfen, die Eltern aber sterben, ermordet werden wie in tragischen Geschichten Überlebender des Konzentrationslagers, wie im Simplizius Simplizissimus Grimmelshausens, wie bei Kindern von Eltern, die in Schande geraten sind. Die Generationenspannung von Verfall und Gedeihen bildet die Folie für die Geschichte der Autorität. Der verfallende Vater - oder allgemeiner der Vater in seiner Schwäche, die Mutter in ihrer Schwäche - schafft ein ungeheures Konfliktfeld für die literarischen Protagonisten in Tochter- und Sohnesrolle. Dies ist für die Literatur des 20. Jhs. besonders prominent und bildet ausserdem inzwischen 
ein psychotherapeutisches Klischee. Kafka gestaltet lange vor Dürrenmatt und lange vor der autobiographischen Abrechnungsliteratur der 70er Jahre, im sprichwörtlichen »Urteil«, einen väterlichen Riesen und Prothesengott in bizarrer Willkür, der den Sohn nicht zum Mann und zum Liebenden werden lässt, sondern als altes Kind verhöhnt und in den Tod treibt.

\section{Im Liebesnest zugrunde gehen}

Dürrenmatt skizziert in seinem Romanentwurf »Der Rebell« einen fürchterlichen und lächerlichen, ungreifbaren und todbringenden Vater im Gewand eines kirchlichen Würdenträgers, der den Sohn verrät und ans Messer liefert. Er erzählt vom einsamen und verlassenen Sohn, dem man auf lächerliche Art eine messianische Maske aufklebt, ein Sohn, der ebensowenig wie Kafkas Verurteilter zum Mann und zum Liebenden werden kann und als altes Kind im Kerkerloch verschmachtet. Aber die Geschichte von der vergeblichen und tödlichen Vatersuche ist nur das eine. Von besonderem Interesse ist etwas anderes, das uns angesichts der effektvollen und eigentümlichen Vater-Sohn-Verstrickung entgehen könnte, in Wirklichkeit aber die Logik dieser Dichtung ausmacht: die Mutter-SohnVerstrickung. Die Dürrenmattsche Todeslust im ausweglosen Labyrinth unter kaltem und heiligem Firmament basiert auf einer Mutter-Sohn-Dramaturgie, die sich der ödipalen Verwicklung, wie Freud sie literarisch analysiert und auf existentielle Glücks- und Katastrophenerfahrungen bezogen hat, exemplarisch gegenüberstellt. In der Tragödie des Sophokles scheitert der starke Sohn an seiner Stärke und findet durch Selbstkastration den Weg zur Verwandlung. In der Romankomödie Dürrenmatts feiert der schwache Sohn seine Schwäche und zelebriert die Selbstauslöschung im mütterlichen Labyrinth. Im Drehbuch ist verdeckt ein anarchisches Mutter-SohnLiebesnest angelegt. Was sind die Glücksbedingungen eines solchen Liebesnestes? Sie lassen sich in allgemeiner Form genau beschreiben: Jenseits des Gesetzes, jenseits der Ordnungsmacht hat die Liebeslust der beiden, Mutter und Sohn, die Chance, sich ins Schrankenlose zu entwickeln, in ein Alles-ist-möglich, in ein seliges lust- und schmerzerfülltes Reich des Polymorph-Perversen, wie Freud sich ausgedrückt hätte. Gleichzeitig handelt es sich um ein Paar, das asymmetrisch einander zugeordnet ist, es gibt notwendig ein Oben und Unten. Der Sohn befindet sich der Mutter gegenüber in der 
unterlegenen Position. Sie ist es, die ihm das Leben gegeben hat, sie ist die Meisterin der Liebe, sie führt ihn in die Liebe ein, sie ist diejenige, die sein Leben organisiert und geschützt hat, sie ist die Nährerin und Ressourcenträgerin. Das Liebesglück zwischen Mutter und Sohn im Liebesnest bedeutet: Sie nährt, schützt und vergöttert ihn. Er überlässt sich ihr. Es gibt keinen Zukunftshorizont, keine Öffnung nach aussen. Es gibt nur völliges Genügen aneinander, völliges Aufgehen im Hier und Jetzt, Bereitschaft, den Augenblick zu leben und danach zu verlöschen, zu sterben im Augenblick des Ineinandersinkens. Es ist die mächtige Mutter, die Grosse Mutter, die den Sohn umfängt, in sich hineinnimmt, der sich der Sohn hingibt, die er mit seinen erotischen Mitteln beglückt. Die Hingabe des Sohnes an die Grosse Mutter ist absolute Preisgabe, es gibt keinen Halt und kein Halten, keinen Schutz und keine Sicherung. Die Mutter kann sich jederzeit der Sexualität, der Phallizität des Sohnes bemächtigen, sie sich aneignen, zum Verschwinden bringen. Er ist ihr ausgeliefert. Er ist, auch wenn sie ihn vergöttert, pflegt und schützt, in einer Position der Schwäche. Er ist sich seiner Männlichkeit, seiner Phallizität nicht sicher, kann sie nicht zu seiner eigenen machen.

Dürrenmatt braucht das Handlungs- und Gefühlspotential der Mutter-Sohn-Intimität als geheime Basis für die Schlüssigkeit der tödlichen Gefangenschaft des Jünglings im Labyrinth, aber er spielt sie, im Unterschied zu späteren Autoren und Filmschaffenden, nicht aus. Die Dynamik wirkt, bleibt selbst aber stumm. Der Sohn figuriert lediglich als stummer Zeuge angesichts eines stumm wirkenden Berührungstabus. Er vergeht nicht im seligen Liebeslabyrinth, sondern wird verlassen, wird zum letzten Hüter des Hauses und begibt sich auf die Suche nach dem Vater. Es ist freilich folgerichtig, dass er diesen Exodus in der Position der Schwäche - aus einer Situation des Preisgegebenseins heraus - unternimmt. So wird er zum Gespött des Vaters. Der lässt den Sohn fallen, macht ihn, den kleinen Gefährten der Mutter, der in Abwesenheit des Vaters im gemachten Nest sass, verächtlich. Der Sohn nimmt die väterliche Verachtungsstrafe als Selbstmarginalisierung und Selbstverachtung, als Bereitschaft zur Selbstauslöschung an. Denn mächtig und furchtbar bleibt das Bild der Mutter. Sie hat die Ferne zum Vater hergestellt, auch dieser ist nur ihr Diener und Priester. Gerade weil und indem sie entindividualisiert, depersonifiziert - kein Gesicht und keine menschliche Stimme hat, herrscht sie als mythische Wesenheit. 


\section{Kein Entkommen}

In der ödipalen Dramaturgie, wie sie Freud am Modell der Sophokleischen Tragödie entwickelt hat, verhält es sich so: Wer mit der Mutter verkehrt und den Vater erschlägt, der ist ein Schadensbringer und wird aus der Gesellschaft ausgeschlossen. Er hat die Chance zur Verwandlung über die Selbstbestrafung - Bild der Blendung bei Sophokles - und erfährt das Leben wissend als ein Gezeichneter. In der Mutter-Sohn-Dramaturgie, wie Dürrenmatt sie anlegt, verhält es sich so: Der Vater zieht sich aus der Mutter-SohnGemeinschaft zurück und überlässt das Feld der Liebe dem MutterSohn-Paar. Mutter und Sohn erkennen einander, genügen einander, etablieren jenseits der sozialen Ordnung eine abgeschlossene Nische bei Preisgabe von Autonomie, Zukunft und Fruchtbarkeit. Die erschlichene ödipale Privilegierung verfält der Verachtung und mündet in Selbstpreisgabe und Tod. Diese Dynamik gestaltet Dürrenmatt in zahlreichen Texten, eine machtvolle überlegene sexuelle Muttergestalt erscheint bemächtigend, verfürerisch und bedrohlich schon in "Grieche sucht Griechin", im Gewand der Rachegöttin inszeniert sie sich im "Besuch der alten Dame«, nicht verführerisch, aber bemächtigend, ausbeutend und in Gefangenschaft haltend zeigt sie sich in den "Physikern" als Anstaltsärztin. Als Schreckbild taucht sie zweifach, als ausliefernde Mutter und verführende Tochter, in "Vinter" auf. Dürrenmatt kennt das Heilige sehr wohl. Es ist die verwandelte Mutter: der indifferent ewige Kosmos, die indifferente Natur, die den Tod bringen kann, wann immer sie will. Das Drehbuch sieht Erhabenheit vor. Da herrscht heilige Scheu. Heiliger Schrecken. Das Weibliche ist gross und mächtig und furchtbar für den Mann, der seiner frontal und unverstellt ansichtig wird. Und gross und furchtbar ist das Weibliche auch, wenn es sich begehrend und im Liebesverlangen dem Sohn nähert. Da bleibt nur ein Rettesich-wer-kann, so lange noch Zeit ist. Sonst muss man dran glauben und alles hergeben, das Leben im Bedarfsfall eingerechnet wie all die Gatten und der abtrünnige Liebhaber im "Besuch der alten Dame«, wie der verirrte Jüngling in "Das Haus" (Dürrenmatt 1998a, 113130), wie der entsetzte Vinter aus der gleichnamigen Erzählung (Dürrenmatt 1998a, 189-232), wie die »Physiker" (Dürrenmatt 1962), die sich im weiblich kontrollierten Schoss der Anstalt so sicher glaubten, wie Moses Melker in »Durcheinandertal« (Dürrenmatt 1989), dessen stationär gebettete, gefrässige Gattin alles weiss, 
alles beherrscht. Da bleibt die religiöse Entrückung des Weiblichen ins indifferent-fern-Unendliche, dort, in den kalten gestirnten Himmel, die "Weltallkälte» (Dürrenmatt 1989, "Mondfinsternis«, 253) zu blicken und zu beten, dass sie fern bleibt, kalt und uninteressiert. Die Einschüchterung schafft die Notwendigkeit der Distanz. Die Distanz verknüpft sich mit der Inszenierung von Lächerlichkeit. Der Mensch in seiner Urlächerlichkeit stellt seinen gefährdeten, bedrohten, beeinträchtigten, geschundenen, von Hässlichkeit und Deformation gezeichneten Prothesenleib dar, offensiv, um ein Liebesbegehren im Keim abzutöten, um die Vereinigung der Leiber zu verhindern. Die aus der Inszenierung von Verachtung und Selbstverachtung gewonnene Isolation hebt aber gerade die existentielle Abhängigkeit des lebendigen Geschöpfes nicht auf, und daher verbindet sich mit der destruktiven Sezierlust die Ergebung. Das ist eine prominente und literarisch-kulturelle Gestaltung des Todestriebes als heilige Todeslust, die in der Gegenwart des 20. Jhs. kräftig wirkt.

\section{Literatur}

B. Boothe, Vom Verlassen des Elternhauses. Die Dramaturgie der Trennung in literaturwissenschaftlicher Perspektive (Buchbesprechung P. von Matt, Verkommene Söhne, missratene Töchter. Familiendesaster in der Literatur, München 1995), Psychotherapie und Sozialwissenschaft 1, 1999, 162-168.

F. Dürrenmatt, Der Besuch der alten Dame, Zürich 1956. Ders., Die Physiker, Zürich 1962. Ders., Romulus der Grosse, Zürich 1964. Ders., Play Strindberg. Totentanz nach August Strindberg, Zürich 1969. Ders., Grieche sucht Griechin. Eine Prosakomödie, Zürich (o.J.). Ders., Der Mitmacher. Ein Komplex, Zürich 1986. Ders., Durcheinandertal, Zürich 1989. Ders., Labyrinth. Stoffe I-III. Der Winterkrieg in Tibet. Mondfinsternis. Der Rebell, Zürich 1998. Ders., Mondfinsternis, XXX. Ders., Turmbau. Stoffe IV-IX. Begegnungen. Querfahrt. Die Brücke. Das Haus. Vinter. Das Hirn, Zürich 1998.

S. Freud, Jenseits des Lustprinzips. Gesammelte Werke XIII, 1920, 1-69. Ders., Das ökonomische Problem des Masochismus. Gesammelte Werke XIII, 1924, 369-383.

H. Kohut, Narzissmus, Frankfurt 1971.

M. Proust, Auf der Suche nach der verlorenen Zeit I. In Swanns Welt. Erster Teil: Combray, Frankfurt 1955 (1913).

F. Schiller, Sämtliche Werke. Säkular-Ausgabe, hg. v. E. von der Hellen, Stuttgart 1904-1905. Ders., Über die tragische Kunst, in: Vom Pathetischen und Erhabenen. Ausgewählte Schriften zur Dramentheorie, hg. v. K.L. Berghahn. Stuttgart 1970, 30-54.

P. von Matt, Verkommene Söhne, missratene Töchter. Familiendesaster in der Literatur, München 1995. Ders., Die Liebe in der Literatur. Zur Dramaturgie einer Himmelsmacht. Familiendynamik 24, 1999, 369-381.

- Dr. Brigitte Boothe ist Professorin für Klinische Psychologie an der Universität Zürich und Mitglied der Leitung des Zürcher Kompetenzzentrums Hermeneutik (ZKH). 